\title{
Mapping memory binding onto the connectome's temporal dynamics: toward a combined biomarker for Alzheimer's disease
}

\author{
Agustin Ibanez ${ }^{1,2,3,4 *}$ and Mario A. Parra ${ }^{5,6}$ \\ 1 Laboratory of Experimental Psychology and Neuroscience (LPEN), Institute of Cognitive Neurology (INECO), Favaloro University, Buenos Aires, Argentina \\ ${ }^{2}$ Laboratory of Cognitive and Social Neuroscience, UDP-INECO Foundation Core on Neuroscience, Diego Portales University, Santiago, Chile \\ ${ }^{3}$ National Scientific and Technical Research Council, Buenos Aires, Argentina \\ ${ }^{4}$ Universidad Autónoma del Caribe, Barranquilla, Colombia \\ ${ }^{5}$ Psychology, Human Cognitive Neuroscience and Centre for Cognitive Ageing and Cognitive Epidemiology, University of Edinburgh, Edinburgh, UK \\ ${ }^{6}$ Alzheimer Scotland Dementia Research Centre, Scottish Dementia Clinical Research Network, Edinburgh, UK \\ *Correspondence: aibanez@ineco.org.ar
}

Edited by:

Ryouhei Ishii, Osaka University Graduate School of Medicine, Japan

Reviewed by:

Masafumi Yoshimura, Kansai Medical University, Japan

Keywords: Alzheimer disease, connectome, EEG/MEG, biomarkers, early detection, mild cognitive impairment

The classical amyloid cascade hypothesis of Alzheimer's disease (AD) has driven research and clinical practice for several decades. It states that the deposition of the amyloid- $\beta$ peptide in the brain parenchyma initiates a sequence of events that ultimately lead to atrophy and $\mathrm{AD}$ dementia. This proposal stimulated the study of specific brain regions mapped along the neurodegeneration sequence (e.g., hippocampus) and their associated impaired functions (e.g., episodic memory). Although anticipated by Mesulam more than 20 years ago (e.g., Mesulam and Asuncion Moran, 1987), it was not until recently that this view has started to change, largely due to the disappointing results of trials relying on the beta-amyloid cascade hypothesis and its variants, e.g., the synaptic beta-amyloid hypothesis.

A new approach based on neuroplasticity of neural networks has shifted the attention from one region to the orchestration of several brain hubs. The connectivity account would play an important role in revealing the transneural spread of misfolded proteins through neural networks in neurodegenerative disease (Pievani et al., 2011; Ibanez and Manes, 2012), and specifically in AD (Raj et al., 2012). In line with this view, the metabolism hypothesis ( $\mathrm{MH}$ ) has been proposed, which suggests that changes in the default mode network (DMN, the ongoing low-frequency fluctuations during resting state between the anterior and posterior cingulate cortex as well as the precuneus) stimulate an activitydependent or metabolism-dependent cascade that promotes the development of the $\mathrm{AD}$ pathology (Buckner et al., 2005). Notably, hyperactive neurons are observed near amyloid plaques in animal models (Busche et al., 2008) and in humans, connectivity hubs overlap the anatomy of A- $\beta$ deposition (Buckner et al., 2009). Abnormal DMN activity discriminates between Mild Cognitive Impairment (MCI), AD and controls (Rombouts et al., 2005; Petrella et al., 2011; Seo et al., 2013; Wang et al., 2013b), and predicts AD conversion (Pievani et al., 2011). Thus, default connectivity seems to be a promising approach to reveal novel mechanisms leading to $\mathrm{AD}$.

However, the DMN seems to be affected by many other diseases (Sonuga-Barke and Castellanos, 2007; Whitfield-Gabrieli and Ford, 2012). Moreover, although the altered DMN might interrupt or affect the brain dynamic in $\mathrm{AD}$ patients, it actually reflects a resting state activity unlikely to explain, on its own, profiles of cognitive decline in $\mathrm{AD}$. The combined analysis of brain connectivity associated with specific cognitive processes affected by AD early in its course with resting DMN is an unexplored area that could help overcome these limitations. This approach may reveal markers for the early or even preclinical detection of neurocognitive impairments in AD. A potential strategy would be to assess the neural connectivity associated with episodic memory tasks (Wang et al., 2013a). However, these tasks have only detected AD-related changes in its prodromal or clinical stages (Fields et al., 2011). A recently developed methodology, namely short-term memory binding (STMB, Parra et al., 2009, 2010, 2011), is intrinsically related to brain networks activation, and appears to be more promising for the preclinical detection of $\mathrm{AD}$. Binding functions, as originally investigated in perception, require a large-scale network integration mechanism (Varela et al., 2001). In AD research, emerging evidence suggests that binding impairments occur at the short term memory level. STMB is a cognitive function responsible for retaining, on a temporary basis, intra-item features thus contributing to the formation of objects' identity. It has been recently assessed with a change detection tasks, which ask participants to judge whether the content of two consecutive arrays of shapes, colors or shape-color combinations is the same or different. STMB is impaired early in AD (Parra et al., 2009) and also in preclinical familial AD (Parra et al., 2010, 2011), preserved in healthy aging (Brockmole et al., 2008), and declines earlier in AD than in other dementias (Della Sala et al., 2012). Moreover, STMB seems to be indexing a pre-hippocampal phase of $\mathrm{AD}$ (Reiman et al., 2010) and recruits other regions (Parra et al., 2014). Thus, a combination of 
both neural (DMN) and cognitive (STMB) integration processes may contribute an early and specific marker of $\mathrm{AD}$ progression. A new research agenda linking resting brain dynamics (DMN) with an active task which (1) relies on neural network integration and (2) is highly specific and sensitive for $\mathrm{AD}$, would represent a powerful approach to the early detection of $\mathrm{AD}$. Importantly, the resting and active connectivity measures drawn from this novel approach can be tracked both with neuroimaging (fMRI) and electromagnetic methods (EEG and MEG).

STMB tasks are well suited to be tracked with EEG or MEG measurement (Luria and Vogel, 2011; Wilson et al., 2012). Typically, the temporal dynamic of the integrative functions assessed by this task would be in the order of milliseconds. These techniques can capture the evoked responses and their neural connections during the whole process of STMB. This task also provides several advantages for EEG/MEG procedures such as high number of trials, stimuli-evoked activity pre and post memory binding process, temporal sequencing, and categories with different levels of difficulty.

Notwithstanding the adequacy of the task's parameters for EEG/MEG recordings, the question of why techniques with low spatial resolution should be considered instead of neuroimaging methods stands out. Several factors support this selection. First, high-density electroencephalography (hd-EEG) and other electromagnetic techniques permit an easy, low-cost, non-invasive, and accessible approach for large-scale multisite studies around the world. Second, high density EEG/MEG technologies have provided an increased spatial resolution of fine temporal dynamics both at the analytical (mathematical methods) and technical (high number of channels, photometry methods, and individual MRI co-recordings) level, making them more suitable to investigate $\mathrm{AD}$-related changes. Third, EEG/MEG techniques have proven useful for characterizing $\mathrm{AD}$ and also for detecting changes in preclinical familial $\mathrm{AD}$ and $\mathrm{MCI}$ (Jackson and Snyder, 2008; Stam, 2010). For example, source EEG functional network disruption in $\mathrm{AD}$ is associated with cognitive decline (Gianotti et al., 2007; Ishii et al.,
2010) (see (Kurimoto et al., 2008; Hsiao et al., 2013) for similar results in MCI), APOE genotype (Canuet et al., 2012) and differentiates between other dementias (Babiloni et al., 2004). Moreover, loss of interregional synchronization between different functional brain regions also reflects cognitive decline in AD (De Haan et al., 2012). Furthermore, the EEG/MEG based connectivity analysis (EMCA) can also be used to track the effect of medication on AD (Babiloni et al., 2006; Gianotti et al., 2008).

There are other direct advantages of using EMCA. The theoretical frame of interdependence between spontaneous and evoked neuroelectric oscillations in terms of frequency and phase reset has been forwarded earlier (e.g., by Basar' group in the eighties). Nevertheless, over the last 10 years, a real increase in technical and mathematical sophistication in EMCA has produced new research possibilities with practical applications (Basar et al., 2013; Larson-Prior et al., 2013). Regarding brain global properties, current graph theoretical network studies of the brain have shown a self-organized small-world network characterized by a combination of focal connectivity and long-distance connections. Graph theory is one of the most powerful forms of connectivity analysis for AD (Pievani et al., 2011; Tijms et al., 2013), and it can be correctly implemented with EEG/MEG signals (e.g., Stam and Van Straaten, 2012; Barttfeld et al., 2013). Regarding time dynamics, different networks are orchestrated in our brain in time windows of milliseconds and the connectivity within and between them is not a static process. Our brain has rapid rhythms that allow for communication between different regions at several frequencies. The high time-resolution of intracranial signals from EEG sources can be quantified by coherence and phase synchronization, two methods that have proved informative in $\mathrm{AD}$ (Czigler et al., 2006; Knyazeva et al., 2012). Moreover, recent methods provide a better characterization of the physiological signal with better spatial location and provide solutions for classical problems of volume conduction (Pascual-Marqui et al., 2011). They also permit the comparison of spontaneous and stimulus-induced activations and the identification of commonalities between them (Lehmann et al., 2010). Moreover, oscillatory neuronal dynamics in the human brain using connectivity analysis of source estimated event-related synchronization at different frequencies is now an available method (Ishii et al., 2013). Transient momentary events (e.g., thoughts) in electromagnetic signals might be incorporated in temporal chunks of processing (10-100 ms) as quasi-stable brain states (Lehmann et al., 2006). In brief, EMCA can track the brain dynamic of rapid fluctuations (Barttfeld et al., 2014) and also of transient activity during very short periods, especially those supporting binding or transient integration.

Thus, assessing the combination of basal resting state influences together with the ongoing activity during a task and its evoked neural response may allow for investigation and characterization of preclinical AD-related changes in brain dynamics. This novel approach offers new possibilities to better understand the cognitive binding problem in the course of $\mathrm{AD}$ as well as the dynamics of cortical integration. This research proposal also requires tackling several methodological and empirical challenges. Although promising, current methods for combining connectivity measures during ongoing activity and evoked responses do not yet fully capture the single trial dynamics. The potential use of connectivity metrics as predictor of patients' clinical outcome is not well understood at present. No single study using EMCA to assess $\mathrm{AD}$ or MCI patients has combined active and resting recordings and the analysis of source connectivity using individual MRI. We believe this combined approach would disclose unknown AD mechanisms. The research growth in these domains of cognitive neuroscience will offer support to key strategies such as combining STMB and EMCA to provide a neurocognitive marker for the preclinical detection of $\mathrm{AD}$. In support to this proposal, recent neuroimaging studies carried out in cases of preclinical familial $\mathrm{AD}$ have revealed a temporal proximity between the onset or appearance of STMB deficits and amyloid- $\beta$ deposition. By the average age at which amyloid- $\beta$ depositions reach a plateau (Fleisher et al., 2012), STMB deficits become detectable behaviorally (Parra et al., 2010). It is worth noticing 
that these mutation carriers would be otherwise completely asymptomatic. This evidence warrants investigation of the hypothesis of a link between connectivity problems as assessed by STMB and EMCA and neurodegenerative changes in AD. Such research would shed further light into the link, or lack thereof, between amyloid changes, cognition and $\mathrm{AD}$.

\section{ACKNOWLEDGMENTS}

This study was supported by grants CONICYT/FONDECYT Regular (1130920 and 1140114), FONCyT-PICT 2012-0412, FONCyT-PICT 2012- 1309, CONICET and the INECO Foundation. Mario A. Parra work is supported by Alzheimer's Society, Grant \# AS-R42303.

\section{REFERENCES}

Babiloni, C., Binetti, G., Cassetta, E., Cerboneschi, D., Dal Forno, G., Del Percio, C., et al. (2004). Mapping distributed sources of cortical rhythms in mild Alzheimer's disease. Neuroimage 22, 57-67. doi: 10.1016/j.neuroimage.2003.09.028

Babiloni, C., Cassetta, E., Dal Forno, G., Del Percio, C., Ferreri, F., Ferri, R., et al. (2006). Donepezil effects on sources of cortical rhythms in mild Alzheimer's disease. Neuroimage 31, 1650-1665. doi: 10.1016/j.neuroimage.2006.02.015

Barttfeld, P., Amoruso, L., Ais, J., Cukier, S., Bavassi, L., Tomio, A., et al. (2013). Organization of brain networks governed by long-range connections index autistic traits in the general population. J. Neurodev. Disord. 5, 16. doi: 10.1186/1866-19555-16

Barttfeld, P., Petroni, A., Báez, S., Urquina, H., Sigman, M., Cetkovich, M., et al. (2014) Functional connectivity and temporal variability of brain connections in adults with attention deficit/hyperactivity disorder and bipolar disorder. Neuropsychobiology 69, 65-75. doi: $10.1159 / 000356964$

Basar, E., Basar-Eroglu, C., Guntekin, B., and Yener, G. G. (2013). Brain's alpha, beta, gamma, delta, and theta oscillations in neuropsychiatric diseases. Suppl. Clin. Neurophysiol. 62, 19-54. doi: 10.1016/B978-0-7020-5307-8.00002-8

Brockmole, J. R., Parra, M. A., Della Sala, S., and Logie, R. H. (2008). Do binding deficits account for age-related decline in visual working memory? Psychon. Bull. Rev. 15, 543-547. doi: 10.3758/PBR.15.3.543

Buckner, R. L., Sepulcre, J., Talukdar, T., Krienen, F. M., Liu, H., Hedden, T., et al. (2009). Cortical hubs revealed by intrinsic functional connectivity: mapping, assessment of stability, and relation to Alzheimer's disease. J. Neurosci. 29, 1860-1873. doi: 10.1523/JNEUROSCI.5062-08.2009

Buckner, R. L., Snyder, A. Z., Shannon, B. J., Larossa, G., Sachs, R., Fotenos, A. F., et al. (2005). Molecular, structural, and functional characterization of Alzheimer's disease. J. Neurosci. 25, 7709-7717. doi: 10.1523/JNEUROSCI.217705.2005
Busche, M. A., Eichhoff, G., Adelsberger, H., Abramowski, D., Wiederhold, K. H., Haass, C., et al. (2008). Clusters of hyperactive neurons near amyloid plaques in a mouse model of Alzheimer's disease. Science 321, 1686-1689. doi: 10.1126/science.1162844

Canuet, L., Tellado, I., Couceiro, V., Fraile, C., Fernandez-Novoa, L., Ishii, R., et al. (2012). Resting-state network disruption and APOE genotype in Alzheimer's disease. PLoS ONE 7:e46289. doi: 10.1371/journal.pone.0046289

Czigler, B., Csikos, D., Gaal, A. Z., Csibri, E., Kiss, E., Hidasi, Z., et al. (2006). [Quantitative EEG analysis in Alzheimer's disease: spectral, coherence and complexity parameters]. Psychiatr. Hung. 21, 300-312.

De Haan, W., Van Der Flier, W. M., Koene, T., Smits, L. L., Scheltens, P., and Stam, C. J. (2012). Disrupted modular brain dynamics reflect cognitive dysfunction in Alzheimer's disease. Neuroimage 59, 3085-3093. doi: 10.1016/j.neuroimage.2011.11.055

Della Sala, S., Parra, M. A., Fabi, K., Luzzi, S. and Abrahams, S. (2012). Short-term memory binding is impaired in $\mathrm{AD}$ but not in nonAD dementias. Neuropsychologia 50, 833-840. doi: 10.1016/j.neuropsychologia.2012.01.018

Fields, J. A., Ferman, T. J., Boeve, B. F., and Smith, G. E. (2011). Neuropsychological assessment of patients with dementing illness. Nat. Rev. Neurol. 7, 677-687. doi: 10.1038/nrneurol.2011.173

Fleisher, A. S., Chen, K., Quiroz, Y. T., Jakimovich, L. J., Gomez, M. G., Langois, C. M., et al. (2012). Florbetapir PET analysis of amyloid-beta deposition in the presenilin 1 E280A autosomal dominant Alzheimer's disease kindred: a crosssectional study. Lancet Neurol. 11, 1057-1065. doi: 10.1016/S1474-4422(12)70227-2

Gianotti, L. R., Kunig, G., Faber, P. L., Lehmann, D., Pascual-Marqui, R. D., Kochi, K., et al. (2008). Rivastigmine effects on EEG spectra and three-dimensional LORETA functional imaging in Alzheimer's disease. Psychopharmacology 198, 323-332. doi: 10.1007/s00213-008-1111-1

Gianotti, L. R., Kunig, G., Lehmann, D., Faber, P. L., Pascual-Marqui, R. D., Kochi, K., et al. (2007). Correlation between disease severity and brain electric LORETA tomography in Alzheimer's disease. Clin. Neurophysiol. 118, 186-196. doi: 10.1016/j.clinph.2006.09.007

Hsiao, F. J., Wang, Y. J., Yan, S. H., Chen, W. T., and Lin, Y. Y. (2013). Altered oscillation and synchronization of default-mode network activity in mild Alzheimer's disease compared to mild cognitive impairment: an electrophysiological study. PLoS ONE 8:e68792. doi: 10.1371/journal.pone.0068792

Ibanez, A., and Manes, F. (2012). Contextual social cognition and the behavioral variant of frontotemporal dementia. Neurology 78, 1354-1362. doi: 10.1212/WNL.0b013e3182518375

Ishii, R., Canuet, L., Aoki, Y., Ikeda, S., Hata, M., Iwase, M., et al. (2013). Non-parametric permutation thresholding for adaptive nonlinear beamformer analysis on MEG revealed oscillatory neuronal dynamics in human brain. Conf. Proc. IEEE Eng. Med. Biol. Soc. 2013, 4807-4810. doi: 10.1109/EMBC.2013.6610623

Ishii, R., Canuet, L., Kurimoto, R., Ikezawa, K., Aoki, Y., Azechi, M., et al. (2010). Frontal shift of posterior alpha activity is correlated with cognitive impairment in early Alzheimer's disease. Psychogeriatrics 10, 138-143. doi: 10.1111/j.14798301.2010.00326.x

Jackson, C. E., and Snyder, P. J. (2008). Electroencephalography and event-related potentials as biomarkers of mild cognitive impairment and mild Alzheimer's disease. Alzheimers Dement. 4, S137-S143. doi: 10.1016/j.jalz.2007.10.008

Knyazeva, M. G., Carmeli, C., Khadivi, A., Ghika, J., Meuli, R., and Frackowiak, R. S. (2012). Evolution of source EEG synchronization in early Alzheimer's disease. Neurobiol. Aging 34, 694-705. doi: 10.1016/j.neurobiolaging.2012.07.012

Kurimoto, R., Ishii, R., Canuet, L., Ikezawa, K., Azechi, M., Iwase, M., et al. (2008). Event-related synchronization of alpha activity in early Alzheimer's disease and mild cognitive impairment. Neurosci. Lett. 443, 86-89. doi: 10.1016/j.neulet.2008.07.015

Larson-Prior, L. J., Oostenveld, R., Della Penna, S., Michalareas, G., Prior, F., Babajani-Feremi, A., et al. (2013). Adding dynamics to the Human Connectome Project with MEG. Neuroimage 80, 190-201. doi: 10.1016/j.neuroimage.2013.05.056

Lehmann, D., Faber, P. L., Gianotti, L. R., Kochi, K., and Pascual-Marqui, R. D. (2006). Coherence and phase locking in the scalp EEG and between LORETA model sources. J. Physiol. Paris 99, 29-36. doi: 10.1016/j.jphysparis.2005.06.005

Lehmann, D., Pascual-Marqui, R. D., Strik, W. K., and Koenig, T. (2010). Core networks for visual-concrete and abstract thought content. Neuroimage 49, 1073-1079. doi: 10.1016/j.neuroimage.2009.07.054

Luria, R., and Vogel, E. K. (2011). Shape and color conjunction stimuli are represented as bound objects in visual working memory. Neuropsychologia 49, 1632-1639. doi: 10.1016/j.neuropsychologia.2010.11.031

Mesulam, M. M., and Asuncion Moran, M. (1987). Cholinesterases within neurofibrillary tangles related to age and Alzheimer's disease. Ann. Neurol. 22, 223-228. doi: 10.1002/ana.410220206

Parra, M. A., Abrahams, S., Fabi, K., Logie, R., Luzzi, S., and Della Sala, S. (2009). Short-term memory binding deficits in Alzheimer's disease. Brain 132, 1057-1066. doi: 10.1093/brain/awp036

Parra, M. A., Abrahams, S., Logie, R. H., Mendez, L. G., Lopera, F., and Della Sala, S. (2010). Visual short-term memory binding deficits in familial Alzheimer's disease. Brain 133, 2702-2713. doi: 10.1093/brain/awq148

Parra, M. A., Della Sala, S., Logie, R. H., and Morcom, A. M. (2014). Neural correlates of shape-color binding in visual working memory. Neuropsychologia 52, 27-36. doi: 10.1016/j.neuropsychologia.2013.09.036

Parra, M. A., Sala, S. D., Abrahams, S., Logie, R. H., Mendez, L. G., and Lopera, F. (2011). Specific deficit of colour-colour short-term memory binding in sporadic and familial Alzheimer's disease. Neuropsychologia 49, 1943-1952. doi: 10.1016/j.neuropsychologia.2011.03.022

Pascual-Marqui, R. D., Lehmann, D., Koukkou, M., Kochi, K., Anderer, P., Saletu, B., et al. (2011). Assessing interactions in the brain with exact lowresolution electromagnetic tomography. Philos. Trans. A Math. Phys. Eng. Sci. 369, 3768-3784. doi: 10.1098/rsta.2011.0081 
Petrella, J. R., Sheldon, F. C., Prince, S. E., Calhoun, V. D., and Doraiswamy, P. M. (2011). Default mode network connectivity in stable vs progressive mild cognitive impairment. Neurology 76, 511-517. doi: 10.1212/WNL.0b013e31820af94e

Pievani, M., De Haan, W., Wu, T., Seeley, W. W., and Frisoni, G. B. (2011). Functional network disruption in the degenerative dementias. Lancet Neurol. 10, 829-843. doi: 10.1016/S1474-4422(11)70158-2

Raj, A., Kuceyeski, A., and Weiner, M. (2012). A network diffusion model of disease progression in dementia. Neuron 73, 1204-1215. doi: 10.1016/j.neuron.2011.12.040

Reiman, E. M., Langbaum, J. B., and Tariot, P. N. (2010). Alzheimer's prevention initiative. Biomark. Med. 4, 3-14. doi: 10.2217/bmm.09.91

Rombouts, S. A., Barkhof, F., Goekoop, R., Stam, C. J., and Scheltens, P. (2005). Altered resting state networks in mild cognitive impairment and mild Alzheimer's disease. Hum. Brain Mapp. 26, 231-239. doi: 10.1002/hbm.20160

Seo, E. H., Lee, D. Y., Lee, J. M., Park, J. S., Sohn, B. K., Lee, D. S., et al. (2013). Whole-brain functional networks in cognitively normal, mild cognitive impairment, and Alzheimer's disease. PLOS ONE 8:e53922. doi: 10.1371/journal.pone.0053922

Sonuga-Barke, E. J., and Castellanos, F. X. (2007). Spontaneous attentional fluctuations in impaired states and pathological conditions. Neurosci. Biobehav. Rev. 31, 977-986. doi: 10.1016/j.neubiorev.2007.02.005
Stam, C. J. (2010). Use of magnetoencephalography (MEG) to study functional brain networks in neurodegenerative disorders. J. Neurol. Sci. 289, 128-134. doi: 10.1016/j.jns.2009.08.028

Stam, C. J., and Van Straaten, E. C. (2012) The organization of physiological brain networks. Clin. Neurophysiol. 123, 1067-1087. doi: 10.1016/j.clinph.2012.01.011

Tijms, B. M., Wink, A. M., De Haan, W., Van Der Flier, W. M., Stam, C. J., et al. (2013). Alzheimer's disease: connecting findings from graph theoretical studies of brain networks. Neurobiol. Aging 34, 2023-2036. doi: 10.1016/j.neurobiolaging.2013.02.020

Varela, F., Lachaux, J. P., Rodriguez, E., and Martinerie, J. (2001). The brainweb: phase synchronization and large-scale integration. Nat. Rev. Neurosci. 2, 229-239. doi: 10.1038/35067550

Wang, L., Li, H., Liang, Y., Zhang, J., Li, X., Shu, N., et al. (2013a). Amnestic mild cognitive impairment: topological reorganization of the defaultmode network. Radiology 268, 501-514. doi: 10.1148/radiol.13121573

Wang, Y., Risacher, S. L., West, J. D., Mcdonald, B. C., Magee, T. R., Farlow, M. R., et al. (2013b). Altered default mode network connectivity in older adults with cognitive complaints and amnestic mild cognitive impairment. J. Alzheimers Dis. 35, 751-760. doi: 10.3233/JAD-130080

Whitfield-Gabrieli, S., and Ford, J. M. (2012). Default mode network activity and connectivity in psychopathology. Annu. Rev. Clin. Psychol. 8, 49-76. doi: 10.1146/annurev-clinpsy-032511143049

Wilson, K. E., Adamo, M., Barense, M. D., and Ferber, S. (2012). To bind or not to bind. J. Vis. 12, 14 doi: $10.1167 / 12.8 .14$

Conflict of Interest Statement: The authors declare that the research was conducted in the absence of any commercial or financial relationships that could be construed as a potential conflict of interest.

Received: 21 January 2014; paper pending published: 19 March 2014; accepted: 01 April 2014; published online: 22 April 2014.

Citation: Ibanez A and Parra MA (2014) Mapping memory binding onto the connectome's temporal dynamics: toward a combined biomarker for Alzheimer's disease. Front. Hum. Neurosci. 8:237. doi: 10.3389/ fnhum.2014.00237

This article was submitted to the journal Frontiers in Human Neuroscience.

Copyright (c) 2014 Ibanez and Parra. This is an openaccess article distributed under the terms of the Creative Commons Attribution License (CC BY). The use, distribution or reproduction in other forums is permitted, provided the original author(s) or licensor are credited and that the original publication in this journal is cited, in accordance with accepted academic practice. No use, distribution or reproduction is permitted which does not comply with these terms. 